\title{
Interactions between polymorphisms in the 3 'untranslated region of the cyclin dependent kinase 6 gene and the human papillomavirus infection, and risk of cervical precancerous lesions
}

\author{
XINGGUANG YE $^{1^{*}}$, LIPENG JING $^{1^{*}}$, XINGMING ZHONG ${ }^{2 *}$, DI XIAO ${ }^{1}$, MEILING OU $^{1}$, \\ CONGCONG GUO $^{1}$, GUANG YANG ${ }^{3}$, CHUNXIA JING $^{1}$ and XIANGCAI WEI ${ }^{1,2}$ \\ ${ }^{1}$ Department of Epidemiology, School of Medicine, Jinan University, Guangzhou, Guangdong 510632; \\ ${ }^{2}$ Family Planning Research Institute of Guangdong, Guangzhou, Guangdong 510062; ${ }^{3}$ Department of \\ Parasitology, School of Medicine, Jinan University, Guangzhou, Guangdong 510632, P.R. China
}

Received January 10, 2017; Accepted March 30, 2017

DOI: $10.3892 / b r .2017 .898$

\begin{abstract}
The human papillomavirus (HPV) infection is essential for the development of cervical cancer and its precursor lesions. However, only certain persistently infected individuals develop cervical cancer. Cyclin-dependent kinase 6 (CDK6) is a critical regulatory cancer-associated gene in the cell cycle and in tumorigenesis. Single nucleotide polymorphisms (SNPs) in microRNA sites in the 3'-untranslated region (UTR) of target genes may result in target gene expression level changes and susceptibility to diseases, including cancer. Therefore, the aim of the present study was to determine whether SNPs in the 3'UTR of the CDK6 gene may affect susceptibility to cervical precancerous lesions in a Chinese population. Five polymorphisms in the 3'UTR of the CDK6 gene were evaluated in 164 cervical precancerous lesion cases and 296 control subjects. Differences in environmental factors between cases and controls were evaluated using the $\chi^{2}$ test or unpaired t-test. Logistic regression was used to examine the association between the five polymorphisms and cervical precancerous lesions. The model-free multifactor dimensionality reduction (MDR) method was performed to evaluate the interaction effect of environment variables and gene polymorphisms. Interactions on the additive scale are calculated by
\end{abstract}

Correspondence to: Professor Xiangcai Wei, Family Planning Research Institute of Guangdong, 17 Meidong Road, Guangzhou, Guangdong 510062, P.R. China

E-mail: dxcwei@163.com

Professor Chunxia Jing, Department of Epidemiology, School of Medicine, Jinan University, 601 Huangpu Road West, Guangzhou, Guangdong 510632, P.R. China

E-mail: jcxphd@gmail.com

*Contributed equally

Key words: cervical precancerous lesions, interaction, single nucleotide polymorphism, cyclin-dependent kinase 6 , micro RNA using the relative excess risk due to interaction (RERI). After controlling for potential confounders, a significantly decreased risk of cervical precancerous lesions for the GA genotype, rs8179, and the AT genotype, rs42033 [GA vs. GA: odds ratio $(\mathrm{OR})_{\text {adjusted }}=0.17,95 \%$ confidence interval (CI), 0.05-0.57; AT vs. AA: $\mathrm{OR}_{\text {adjusted }}=0.18,95 \% \mathrm{CI}, 0.05-0.59$, respectively] was identified. Furthermore, following MDR analysis, a significant three-locus interaction model was identified, which involved the HPV infection, the number of pregnancies and rs8179. Additionally, a significant antagonistic interaction between the HPV infection and rs8179 was identified on an additive scale. Haplotype AGTA was associated with a decreased risk of developing cervical precancerous lesions $\left(\mathrm{OR}_{\text {adjusted }=} 0.21\right.$; 95\% CI, 0.06-0.75). Thus, the present results indicated that the rs8179 and rs42033 polymorphisms confer genetic susceptibility to cervical precancerous lesions. Furthermore, the interaction between the rs8179 polymorphism in CDK6 and the HPV infection and haplotype AGTA may be associated with cervical precancerous lesions.

\section{Introduction}

Cervical cancer is the third most commonly diagnosed type of cancer and fourth leading cause of cancer mortalities in females worldwide (1). Furthermore, with more than half a million new cases and 265,700 mortalities per year, cervical cancer continues to constitute a major public health problem, particularly in developing countries, such as China (2). The development of cervical cancer is a multistep process involving a precursor preinvasive stage (3). Typically, it takes several years, even decades, to progress from pre-cancer to invasive cervical cancer, which offers many opportunities for intervention. Therefore, the elucidation of the molecular pathogenesis of cervical cancer may contribute to reducing the incidence and mortality rates of cervical cancer (4).

The human papillomavirus (HPV) infection is essential for the development of cervical cancer and its precursor lesions $(5,6)$. However, only certain individuals who remain infected with high-risk HPV develop cervical precancerous lesions and cervical cancer. Although HPV is important for 
the transformation of cervical epithelial cells, it is insufficient for the development of cervical cancer, and there are a variety of environmental and heritable genetic conditions that influence the development of cervical cancer $(7,8)$. Accumulating evidence has revealed the marked potential of microRNAs (miRNAs) for treating cervical cancer (9-11).

Various studies have indicated that tumorigenesis may be caused by regulation disorders of cell cycle-associated proteins, including cyclins, cyclin-dependent kinases (CDKs) and CDK inhibitors. CDK6 is a CDK family member located on human chromosome 7. Its activity first appears in the mid- $G_{1}$ phase to phosphorylate, and thus regulate, the activity of tumor suppressor protein retinoblastoma $(\mathrm{Rb})$. By releasing transcription factor, E2 factor $(\mathrm{E} 2 \mathrm{~F})$ into the nucleus, the promoters of associated genes, which mediate tumorigenesis, are affected $(9,12)$. Furthermore, various miRNA components are reported to be involved in CDK6-mediated tumorigenesis, such as miR-145, miR-320 and miR-29 $(9,10,13)$.

miRNAs are a class of small, noncoding RNAs that function as post-transcriptional regulators of gene expression by binding to the 3'-untranslated region (UTR) of target mRNA through a seed-match region, leading to translational repression or cleavage of target mRNA (14). Single-nucleotide polymorphisms (SNPs) located in the 3'-UTRs of genes may affect interactions with microRNAs (miRNAs), whose association with tumorigenesis is currently a focus of research $(14,15)$. One miRNAtargets numerous messenger RNAs (mRNAs), and one mRNA may be regulated by more than one miRNA. Therefore, functional variations, such as SNPs located in the 3'-UTRs of cancer-associated genes, may cause differential regulation of target gene expression and simultaneously alter numerous molecular pathways that are associated with tumorigenesis. However, SNPs of the 3'-UTR region of the CDK6 gene have rarely been investigated.

Under the hypothesis that gene polymorphisms or haplotypes of the CDK6 gene have an impact on cervical precancerous lesions, a case-control study was performed to demonstrate whether SNPs located in the miRNA-binding sites within the 3'-UTR of the CDK6 mRNAs or haplotypes influence the susceptibility of cervical precancerous lesions. Furthermore, the potential interactions between these SNPs and environmental factors in the etiology of cervical precancerous lesions were investigated in a Chinese population.

\section{Materials and methods}

Subjects. The present study was approved by the Ethics Committee of the School of Medicine, Jinan University (Guangzhou, China). Cervical specimens were collected with a Cytobrush (Qiagen, Inc., Valencia, CA, USA) and placed into a ThinPrep Pap test vial (Hologic, Inc., Marlborough, MA, USA) containing $20 \mathrm{ml}$ PreservCyt Solution (Hologic, Inc.). Referral Pap specimens were used for the ThinPrep cytologic test, which was performed by Kingmed Center for Clinical Laboratory Corporation (Guangzhou, China) using the ThinPrep 2000 System (Hologic, Inc.) and evaluated for routine screening cytology. A Pap smear was positive for a squamous intraepithelial lesion (SIL) if there was a low (LSIL) or high (HSIL) grade SIL, as classified according to the Bethesda Classification System (16). Excluding cases with other types of uterine disease and without a history of hysterectomy, a total of 164 cases [120 LSIL (73.2\%) and 44 HSIL (26.8\%)] were recruited. A total of 296 control samples without an intraepithelial lesion or malignancy were recruited from the area of residence of the cases. The study was explained to each individual and written consent for their participation was obtained.

HPV testing. Exfoliated cervical cell samples were collected from vaginal swabs between May 2013 and November 2014 and conserved in $2.5 \mathrm{ml}$ denaturation buffer (Qiagen, Inc.). Total DNA from cervical cells was extracted using commercial magnetic beads kit (Chemagen; PekinElmer, Inc., Waltham, MA, USA) according to the manufacturer's instructions. Subsequently, 16 HPV genotypes were detected, including HPV16, HPV18, HPV31, HPV33, HPV35, HPV39, HPV45, HPV51, HPV52, HPV56, HPV58, HPV59, HPV66, and HPV68 (all high risk HPV), as well as HPV6 and HPV11 (low risk HPV), using the MassARRAY (Sequenom, Inc., San Diego, CA, USA) technique based on matrix-assisted laser desorption/ionization time-of flight (MALDI-TOF) mass spectrometry) $(17,18)$. These procedures were performed in the clinical standard laboratory of the Beijing Genomics Institute (Shenzhen, China).

Genomic DNA extraction and genotyping. Genomic DNA was extracted from peripheral whole blood $(5 \mathrm{ml}$; collected from women who attended the Family Planning Service Stations or nearby medical institutions between May 2013 and November 2014) using a TIANamp blood DNA extraction kit (TianGen Biotech Co., Ltd., Beijing, China) according to the manufacturer's instructions. All DNA samples were resuspended in water and stored at $-20^{\circ} \mathrm{C}$ until use. The DNA concentration was determined using a spectrophotometer (Nano Drop ND-1000; PerkinElmer, Inc.). Samples with a mean optical density (OD) $260 \mathrm{~nm} / \mathrm{OD} 280 \mathrm{~nm}$ of $1.8-2.0$ and DNA concentration $>20 \mathrm{ng} / \mu$ l were considered to be free of contamination.

Subsequently, CDK6 gene targeted by miRNAs was identified using miRBase (http://www.mirbase.org/) and TargetScan (http://www.targetscan.org/vert_71/). To ensure the reliability of the predictions, multiple prediction algorithms were applied to assess the binding capacity of miRNAs, including RNA22 (https://cm.jefferson.edu/rna22/Interactive/) and BiBiServ2 (http://bibiserv2.cebitec.uni-bielefeld.de/). SNPs residing on the miRNA-binding sites were found by an extensive search in dbSNP (http://www.ncbi.nlm.nih.gov/SNP) using Haploview (https://www.broadinstitute.org/haploview/haploview) and HapMap (http://hapmap.ncbi.nlm.nih.gov/). Finally, five SNPs (rs8179, rs4272, rs42033, rs42035 and rs2377) were identified in predicted miRNA-binding sites with minor allele frequencies $>0.05$ in the Chinese Han population. The selected SNPs were genotyped using MALDI-TOF within the MassARRAY system) (19). The distribution of SNPs in the CDK6 gene is presented in Table I.

Statistical analysis. A t-test or $\chi^{2}$ test was used to compare case and control subjects for the selected demographic characteristics. The Hardy-Weinberg equilibrium theory $\left(p^{2}+2 p q+q^{2}=1\right.$; where $p$ is the frequency of the wild-type allele and $q$ is 
Table I. Distribution of SNPs in the CDK6 gene.

\begin{tabular}{lccccc}
\hline SNP ID & Position & $\begin{array}{c}\text { Functional } \\
\text { region }\end{array}$ & $\begin{array}{c}\text { Major/minor } \\
\text { alleles }\end{array}$ & MAF, \% & $\begin{array}{c}\text { P-value (HWE) } \\
\text { (control) }\end{array}$ \\
\hline $\begin{array}{l}\text { CDK6 (7q21.2) } \\
\text { rs8179 }\end{array}$ & 92606850 & 3'-UTR & G/A & 3.4 & 0.41 \\
rs4272 & 92607515 & 3'-UTR & A/G & 15.3 & 1.00 \\
rs42033 & 92608219 & 3'-UTR & A/T & 3.2 & 0.45 \\
rs42035 & 92610217 & 3'-UTR & T/C & 13.8 & 0.98 \\
rs42377 & 92614358 & 3'-UTR & G/A & 16.3 & 0.96 \\
rs8179 & 92606850 & 3'-UTR & G/A & 3.4 & 0.41 \\
\hline
\end{tabular}

SNP, single nucleotide polymorphism; CDK6, cyclin-dependent kinase 6; MAF, minor allele frequency; HWE, Hardy-Weinberg equilibrium; UTR, untranslated region.

the frequency of the variant allele) was used in controls to calculate the genotype frequencies of all five SNPs using the $\chi^{2}$ test. In addition, logistic regression was used to calculate the odds ratios (ORs) and their relative 95\% confidence intervals (CIs) for risk estimation. A $\chi^{2}$ test was also used to evaluate the dependence of the allele frequencies between the case and control subjects.

The multifactor dimensionally reduction (MDR) approach $(20,21)$ was used to evaluate high-order gene-gene and gene-environment interactions in cervical precancerous lesions risk. To establish the best $\mathrm{n}$-factor model, the data were divided into 10 sets: One for testing and nine for training. Briefly, multilocus genotypes were pooled into high- and low-risk groups, reducing the genotype predictors to one dimension. An MDR permutation text procedure was used to evaluate the significance of the selected models by calculating empirical 1,000-fold permutation tests. The best prediction model was selected based on maximum testing balance accuracy (TBA) and cross-validation consistency (CVC). MDR results were considered to be statistically significant at $\mathrm{P}<0.05$. Based on the MDR results, the interactions of significance were selected for the logistic model to calculate the OR.

The additive interaction between the factors was subsequently evaluated according to the following strategy (22). Among case and control subjects, a binary classification was used for the HPV infection (infection vs. non-infection) and the genotypes (homozygous for the major allele vs. one or two copies of the minor allele). The risk for cervical precancerous lesions for a given SNP and HPV infection status was expressed by ORi, $\mathrm{j}$, where the first index (i) indicated the HPV infection status, coded as 0 for non-infected subjects and 1 for infected subjects, and the second index (j) indicated the SNP genotype, which was coded as 0 for subjects homozygous for the major allele and 1 for subjects bearing one or two copies of the minor allele. Subjects who were HPV-uninfected and homozygous for the major allele served as the reference group, and their cervical precancerous lesion risk was coded as $\mathrm{OR}_{00}=1$. The relative ORs were obtained by logistic regression. The CIs were calculated by the regression coefficients and corresponding covariance matrix (23). Deviation from an additive model was calculated as the relative excess risk due to interaction (RERI). Biological interactions in the regression models were evaluated as departure from additivity. Based on the adjusted ORs obtained in the logistic regression models, an Excel spreadsheet (www.epinet.se) was used to calculate RERI on an additive scale and its corresponding CIs (23). An RERI value $(95 \% \mathrm{CI})$ that does not cross 0 indicates a biological interaction (24). In addition, RERI $>0$ indicates a positive interaction or more than additivity and RERI $<0$ indicates a negative interaction or less than additivity $(25,26)$.

Finally, haplotype analysis (haplotype frequency estimation and linkage dis-equilibrium) between the groups was performed using online SNPStats software (https://www. snpstats.net/snpstats/). The global score test was used to estimate the overall differences in haplotype frequencies between case and control subjects. The estimated adjusted ORs and 95\% CI were also calculated to assess the effect of individual haplotypes on cervical precancerous lesions.

MDR software v.3.0.2 and MDR permutation testing software (version 1.0 beta 2) were used in the current study and were freely available online (www.epistasis.org). All other statistical analyses were performed using SPSS software v.16.0 (SPSS, Inc., Chicago, IL, USA). P $<0.05$ was considered to indicate a statistically significant difference.

\section{Results}

Characteristics of the study population. The frequency distributions of selected demographic characteristics of the case and control subjects are presented in Table II. Increased risk factors for cervical precancerous lesions were observed for a smoking history, shorter time from menarche to the first intercourse (TMI), higher number of pregnancies and HPV infection. No significant differences were observed between case and control subjects in terms of age, body mass index (BMI), age at menarche and age at first intercourse.

Association between SNPs located within the 3'-UTR of the CDK6 gene and cervical precancerous lesion susceptibility. Five SNPs in the 3'-UTR of the CDK6 gene were selected in the current study. The genotype distributions of the five SNPs in the control subjects were in Hardy-Weinberg equilibrium (Table I). As presented in Table III, significant differences 
Table II. Demographic and clinical characteristics of cervical precancerous lesion patients $(\mathrm{n}=164)$ and control subjects ( $\mathrm{n}=296)$.

\begin{tabular}{|c|c|c|c|c|}
\hline & Controls, n (\%) & Cases, n (\%) & OR $(95 \% \mathrm{CI})$ & P-value \\
\hline Age $($ mean $\pm S D)$, years & $42.92 \pm 7.35$ & $41.49 \pm 7.97$ & $\mathrm{~N} / \mathrm{A}$ & $0.053^{\mathrm{a}}$ \\
\hline Body mass index $\left(\mathrm{kg} / \mathrm{m}^{2}\right)$ & $22.44 \pm 3.20$ & $22.14 \pm 2.82$ & N/A & $0.312^{\mathrm{a}}$ \\
\hline \multicolumn{5}{|l|}{ Tobacco smoking } \\
\hline No & $253(85.5)$ & $120(73.2)$ & Ref. & \multirow[t]{2}{*}{$0.001^{\mathrm{b}, \mathrm{c}}$} \\
\hline Yes & $43(14.5)$ & $44(26.8)$ & $2.16(1.34-3.46)$ & \\
\hline Age at menarche (mean $\pm S D)$, years & $14.85 \pm 1.63$ & $15.12 \pm 1.96$ & $\mathrm{~N} / \mathrm{A}$ & $0.113^{\mathrm{a}}$ \\
\hline Age at first intercourse (mean $\pm S D$ ), years & $22.91 \pm 2.89$ & $22.35 \pm 2.92$ & $\mathrm{~N} / \mathrm{A}$ & $0.050^{\mathrm{a}}$ \\
\hline TMI (mean \pm SD), years & $8.05 \pm 3.21$ & $7.23 \pm 3.09$ & N/A & $0.008^{b, c}$ \\
\hline Number of pregnancies (mean $\pm \mathrm{SD}$ ) & $2.48 \pm 1.19$ & $2.77 \pm 1.46$ & $\mathrm{~N} / \mathrm{A}$ & $0.020^{\mathrm{b}, \mathrm{c}}$ \\
\hline \multicolumn{5}{|l|}{ High risk human papillomavirus infection } \\
\hline No & $208(70.3)$ & $32(19.5)$ & Ref. & \multirow[t]{2}{*}{$<0.001^{\mathrm{b}, \mathrm{c}}$} \\
\hline Yes & 88 (29.7) & $132(80.5)$ & $9.75(6.16-15.44)$ & \\
\hline
\end{tabular}

${ }^{\mathrm{a}}$ Two-sided t-test; ${ }^{\mathrm{b}} \mathrm{P}<0.05$, ${ }^{\mathrm{c}}$ Two-sided $\chi^{2}$ test. SD, standard deviation; TMI, the time from menarche to the first intercourse; Ref., reference.

were identified in the rs8179 and rs42033 alleles, as well as the genotype distributions between the case and control subjects. Logistic regression analysis revealed that, following adjustment for HPV infection, age, BMI, tobacco smoking, age at menarche, age at first intercourse and number of pregnancies, the GA genotype in rs8179 was associated with a 0.17 -fold decreased cervical precancerous lesions risk compared with the AA genotype (95\% CI, 0.05-0.57; P=0.004) and the AT genotype in rs42033 was associated with a 0.18 -fold decreased cervical precancerous lesions risk compared with the AA genotype (95\% CI, 0.05-0.59; $\mathrm{P}=0.005)$. While the other three SNPs were not observed to be relevant to the risk of cervical precancerous lesions.

Gene-environment interaction for cervical precancerous lesions susceptibility. According to the MDR selection model, the best model was HPV infection, which had the maximum CVC (10/10) and highest TBA, 75.38\%; significance test, $\mathrm{P}=0.001$; permutation test, $\mathrm{P}=0.000-0.001$. The second was a three-factor interaction model, containing HPV infection, the number of pregnancies and rs8179, with maximum CVC (8/10) and highest TBA, 76.47\%; significance test, $\mathrm{P}=0.001$; permutation test, $\mathrm{P}=0.000-0.001$ ) (Table IV). Additionally, the interactive effects of the HPV infection and each SNP based on an additive scale were evaluated. According to the RERI indexes, a significant antagonistic interaction was identified between CDK6 rs8179, rs42033 and HPV infection (RERI=-9.00, 95\% CI, -14.69 to $-3.30 ; \mathrm{P}=0.002$; RERI=-8.84, $95 \% \mathrm{CI},-14.45$ to $-3.22 ; \mathrm{P}=0.002$ ) as preseted in Table $\mathrm{V}$.

Three-way interaction between HPV infection, rs311678 status and number of pregnancies with the risk for cervical precancerous lesions. Based on the MDR model, a risk analysis of different combinations was performed among the three factors (Table VI). The combination without any risk factors [including non-HPV-infected, wild-type for rs8179 and a reduced number of pregnancies $(<3)$ served as a reference group. The individuals with a combination of three factors had a 10.24-fold greater risk of developing cervical precancerous lesions (95\% CI, 1.25-84.07; $\mathrm{P}=0.030)$, the OR for cervical precancerous lesions in the presence of HPV infection, wild-type for rs8179 and reduced number of pregnancies $(<3)$ was greater $(\mathrm{OR}=12.75 ; 95 \% \mathrm{CI}, 6.03-26.97 ; \mathrm{P}<0.001)$, while the highest $\mathrm{OR}$ for cervical precancerous lesions was in the presence of HPV infection, wild-type for rs8179 and a greater number of pregnancies $(\geq 3)(\mathrm{OR}=20.65$; $95 \% \mathrm{CI}, 9.60-44.44$; $\mathrm{P}<0.001)$.

Haplotype analysis. Strong linkage disequilibrium values were observed between rs8179, rs4272, rs42033 and rs42377 $\left(D^{\prime} \geq 0.86, \mathrm{r}^{2}=0.36-0.90\right.$; Fig. 1). Haplotypes were constructed based on four CDK6 polymorphisms (rs8179, rs4272, rs42033 and rs42377). The overall global test identified a difference in the frequency of haplotypes between case and control subjects for rs8179-rs4272-42033-42377 ( $\mathrm{P}=0.002)$. The results indicated that the haplotype AGTA significantly correlated with a reduced risk of cervical precancerous lesions $(\mathrm{OR}=0.21,95 \%$ CI, 0.06-0.75; $\mathrm{P}=0.016$; Table VII) compared with the highest frequency haplotype GAAG after adjusting for HPV infection, age, BMI, tobacco smoking, age at menarche, age at first intercourse and number of pregnancies. No other frequency difference was observed in these haplotypes between cervical precancerous lesion patients and control subjects.

\section{Discussion}

To the best of our knowledge, this is the first study to investigate the interactive effects of SNPs in the 3'-UTR of the CDK6 gene and HPV infection on the risk of cervical precancerous lesions. To investigate whether SNPs in the CDK6 gene affected the occurrence of cervical precancerous lesions, the associations between variants of the CDK6 gene and cervical precancerous lesions were comprehensively analyzed.

CDK6 is a critical regulatory cancer-associated gene in tumorigenesis and is highly expressed in the majority of cancer types, such as breast, ovarian, gastric, pancreatic and cervical 
Table III. Allele frequencies and genotype distributions of cyclin-dependent kinase 6 SNPs in cervical precancerous lesion patients $(n=164)$ and control subjects $(n=296)$.

\begin{tabular}{|c|c|c|c|c|c|c|c|}
\hline SNP & Variants & Controls, n (\%) & Cases, n (\%) & OR $(95 \% \mathrm{CI})$ & P-value & Oradjusted $(95 \% \mathrm{CI})^{\mathrm{a}}$ & P-value \\
\hline \multirow[t]{2}{*}{ rs8179-Allele } & G & $565(95.4)$ & $324(98.8)$ & 1 (Ref.) & & & \\
\hline & $\mathrm{A}$ & $27(4.6)$ & $4(1.2)$ & $0.26(0.09-0.75)^{\mathrm{b}}$ & $0.007^{\mathrm{b}}$ & & \\
\hline \multirow[t]{3}{*}{ Genotype } & GG & $269(90.9)$ & $160(97.6)$ & 1 (Ref.) & $\mathrm{N} / \mathrm{A}$ & 1 (Ref.) & N/A \\
\hline & GA & $27(9.1)$ & $4(2.4)$ & $0.25(0.09-0.73)^{\mathrm{b}}$ & $0.011^{\mathrm{b}}$ & $0.17(0.05-0.57)^{\mathrm{b}}$ & $0.004^{\mathrm{b}}$ \\
\hline & AA & $0(0.0)$ & $0(0.0)$ & - & - & - & - \\
\hline \multirow[t]{2}{*}{ rs4272-Allele } & A & $501(84.6)$ & $278(84.8)$ & 1 (Ref.) & & & \\
\hline & G & $91(15.4)$ & $50(15.2)$ & $0.99(0.68-1.44)$ & 0.959 & & \\
\hline \multirow[t]{3}{*}{ Genotype } & AA & $212(71.6)$ & $117(71.3)$ & 1 (Ref.) & N/A & 1(Ref.) & N/A \\
\hline & GA & $77(26.0)$ & $44(26.8)$ & $1.04(0.67-1.60)$ & 0.875 & $1.26(0.75-1.12)$ & 0.390 \\
\hline & GG & $7(2.4)$ & $2(1.8)$ & $0.78(0.20-3.06)$ & 0.718 & $0.59(0.12-3.05)$ & 0.532 \\
\hline \multirow[t]{2}{*}{ Recessive } & $\mathrm{AA}+\mathrm{GA}$ & $289(97.6)$ & $161(98.2)$ & 1 (Ref.) & $\mathrm{N} / \mathrm{A}$ & 1 (Ref.) & $\mathrm{N} / \mathrm{A}$ \\
\hline & GG & $7(2.4)$ & $3(1.8)$ & $0.77(0.20-3.02)$ & 0.695 & $0.56(0.11-2.82)$ & 0.478 \\
\hline \multirow[t]{2}{*}{ Dominant } & AA & $212(71.6)$ & $117(71.3)$ & 1 (Ref.) & $\mathrm{N} / \mathrm{A}$ & 1 (Ref.) & $\mathrm{N} / \mathrm{A}$ \\
\hline & $\mathrm{GA}+\mathrm{GG}$ & $84(28.4)$ & $47(28.7)$ & $1.02(0.67-1.55)$ & 0.949 & $1.19(0.72-1.98)$ & 0.501 \\
\hline \multirow[t]{2}{*}{ rs42033-Allele } & A & $567(95.8)$ & $324(98.8)$ & 1 (Ref.) & & & \\
\hline & $\mathrm{T}$ & $25(4.21)$ & $4(1.2)$ & $0.28(0.10-0.81)^{\mathrm{b}}$ & $0.013^{\mathrm{b}}$ & & \\
\hline \multirow[t]{3}{*}{ Genotype } & AA & $271(91.5)$ & $160(97.6)$ & 1 (Ref.) & N/A & 1 (Ref.) & N/A \\
\hline & AT & $25(8.4)$ & $4(2.4)$ & $0.27(0.09-0.79)^{\mathrm{b}}$ & $0.017^{\mathrm{b}}$ & $0.18(0.05-0.59)^{\mathrm{b}}$ & $0.005^{\mathrm{b}}$ \\
\hline & TT & $0(0.0)$ & $0(0.0)$ & - & - & - & - \\
\hline \multirow[t]{2}{*}{ rs42035-Allele } & $\mathrm{T}$ & $508(85.8)$ & $285(86.9)$ & 1 (Ref.) & & & \\
\hline & $\mathrm{C}$ & $84(14.2)$ & $43(13.1)$ & $0.91(0.62-1.36)$ & 0.649 & & \\
\hline \multirow[t]{3}{*}{ Genotype } & $\mathrm{TT}$ & $218(73.7)$ & $122(74.4)$ & 1 (Ref.) & N/A & 1 (Ref.) & N/A \\
\hline & $\mathrm{TC}$ & $72(24.3)$ & $41(25.0)$ & $1.02(0.65-1.59)$ & 0.939 & $0.87(0.51-1.47)$ & 0.591 \\
\hline & $\mathrm{CC}$ & $6(2.0)$ & $1(0.6)$ & $0.30(0.04-2.50)$ & 0.265 & $0.40(0.04-3.86)$ & 0.429 \\
\hline \multirow[t]{2}{*}{ Recessive } & $\mathrm{TT}+\mathrm{TC}$ & $290(98.0)$ & $163(99.4)$ & 1 (Ref.) & N/A & 1 (Ref.) & N/A \\
\hline & $\mathrm{CC}$ & $6(2.0)$ & $1(0.6)$ & $0.30(0.04-2.48)$ & 0.262 & $0.42(0.04-3.99)$ & 0.447 \\
\hline \multirow[t]{2}{*}{ Dominant } & TT & $218(73.7)$ & $122(74.4)$ & 1 (Ref.) & N/A & 1 (Ref.) & N/A \\
\hline & $\mathrm{TC}+\mathrm{CC}$ & $78(26.4)$ & $42(25.6)$ & $0.96(0.62-1.49)$ & 0.862 & $0.83(0.50-1.40)$ & 0.493 \\
\hline \multirow[t]{2}{*}{ rs42377-Allele } & $\mathrm{G}$ & $494(83.4)$ & $276(84.1)$ & 1 (Ref.) & & & \\
\hline & A & 98 (16.6) & 52 (15.9) & $0.95(0.66-1.37)$ & 0.78 & & \\
\hline \multirow[t]{3}{*}{ Genotype } & GG & $206(69.9)$ & $116(70.7)$ & 1 (Ref.) & N/A & 1 (Ref.) & N/A \\
\hline & GA & $82(27.7)$ & $44(26.8)$ & $0.95(0.62-1.47)$ & 0.826 & $1.12(0.66-1.87)$ & 0.682 \\
\hline & AA & $8(2.7)$ & $4(2.4)$ & $0.89(0.26-3.01)$ & 0.849 & $0.77(0.18-3.35)$ & 0.726 \\
\hline \multirow[t]{2}{*}{ Recessive } & $\mathrm{GG}+\mathrm{GA}$ & $288(97.3)$ & $160(97.6)$ & 1 (Ref.) & N/A & 1 (Ref.) & N/A \\
\hline & AA & $8(2.7)$ & $4(2.4)$ & $0.90(0.27-3.04)$ & 0.865 & $0.75(0.17-3.22)$ & 0.693 \\
\hline \multirow[t]{2}{*}{ Dominant } & GG & $206(69.6)$ & $116(70.7)$ & 1 (Ref.) & $\mathrm{N} / \mathrm{A}$ & 1 (Ref.) & $\mathrm{N} / \mathrm{A}$ \\
\hline & $\mathrm{GA}+\mathrm{AA}$ & $90(30.4)$ & $48(29.3)$ & $0.95(0.63-1.44)$ & 0.799 & $1.08(0.65-1.79)$ & 0.764 \\
\hline
\end{tabular}

${ }^{a}$ Adjusted for human papillomavirus infection, age, body mass index $\left(\mathrm{kg} / \mathrm{m}^{2}\right)$, tobacco smoking, age at menarche (years), age at first intercourse (years) and number of pregnancies. ${ }^{b} \mathrm{P}<0.05$. SNP, single nucleotide polymorphism; OR, odds ratio; CI, confidence interval.

cancers (27-31). CDK6 is ubiquitously expressed in human tumor cells, and is involved in cell cycle control and differentiation by promoting the $\mathrm{G}_{1} / \mathrm{S}$ transition $(12,32,33)$. CDK6 regulates the progression of the $G_{1}$ phase by combining with cyclin $\mathrm{D}$ to promote phosphorylation of the tumor suppressor gene, $\mathrm{Rb}(\mathrm{pRb})$, and by releasing transcription factor, E2F, into the nucleus to affect the promoters of the associated genes may affect tumorigenesis (12).

To date, various studies have investigated the association between SNPs in miRNA binding sites and cervical cancer risk, three of which have involved SNPs located in miRNA target genes (i.e., LAMB3-rs2566, BCL2-rs3744935 and 
Table IV. Multifactor dimensionality reduction models of the cyclin-dependent kinase 6 gene and environmental factors of cervical precancerous lesions.

\begin{tabular}{lccr}
\hline Best models & $\begin{array}{c}\text { Training balanced } \\
\text { accuracy }\end{array}$ & $\begin{array}{c}\text { Testing balanced } \\
\text { accuracy }\end{array}$ & $\begin{array}{c}\text { Cross-validation } \\
\text { consistency }\end{array}$ \\
\hline HPV infection & 0.7538 & 0.7538 & $10 / 10$ \\
HPV infection, rs8179 & 0.7615 & 0.7582 & $8 / 10$ \\
HPV infection, rs8179 & 0.7647 & 0.7582 & $8 / 10$ \\
and number of pregnancies & & & $0.000-0.001$ \\
\hline
\end{tabular}

${ }^{\mathrm{a}} 1,000$-fold permutation test. The best model is emboldened. HPV, human papillomavirus.

Table V. Results for gene-environment interaction analysis for each candidate single nucleotide polymorphism and HPV infection.

Deviation from additive model

Interaction group

\begin{tabular}{ll}
\hline 1 & rs42035*HPV infection \\
2 & rs42033*HPV infection \\
3 & rs42377*HPV infection \\
4 & rs4272*HPV infection \\
5 & rs8179*HPV infection
\end{tabular}

Relative excess risk due to

interaction (95\% confidence interval)

P-value ${ }^{\mathrm{a}}$

$$
\begin{aligned}
& -4.64(-11.36 \text { to } 2.08) \\
& -9.00(-14.69 \text { to }-3.30) \\
& -0.09(-7.26 \text { to } 7.09) \\
& 1.04(-6.89 \text { to } 8.99) \\
& -8.84(-14.45 \text { to }-3.22)
\end{aligned}
$$

0.176

0.002

0.981

0.796

0.002

${ }^{a}$ Adjusted for age (years), body mass index $\left(\mathrm{kg} / \mathrm{m}^{2}\right)$, tobacco smoking, age at menarche (years), age at first intercourse (years) and number of pregnancies. Statistically significant values are emboldened. HPV, human papillomavirus.

Table VI. Risk group analysis with three risk factors: HPV infection, number of pregnancies and rs8179.

\begin{tabular}{lcccccc}
\hline HPV infection & Number of pregnancies & rs8179 & Cases & Controls & Odds ratio (95\% confidence interval) & P-value \\
\hline- & - & - & 11 & 106 & 1 & 0.070 \\
- & + & - & 20 & 85 & - & - \\
- & - & + & 0 & 12 & $0.94-4.70)$ \\
- & + & + & 1 & 5 & $1.78(0.19-16.92)$ & 0.616 \\
+ & - & - & 62 & 46 & $2.75(6.03-26.97)$ & $<0.001$ \\
+ & + & - & 67 & 32 & $0.89(0.10-8.10)$ & 0.916 \\
+ & - & + & 1 & 8 & $10.24(1.25-84.07)$
\end{tabular}

${ }^{a}$ Adjusted for age (years), body mass index $\left(\mathrm{kg} / \mathrm{m}^{2}\right)$, tobacco smoking, age at menarche (years), age at first intercourse (years); ${ }^{\mathrm{b}}$ number of pregnancies (where,$-<3$ and,$+ \geq 3$ ). Statistically significant values are emboldened. HPV, human papillomavirus.

TNFAIP8-rs11064) (34-36). These studies indicated that SNPs in the 3'-UTR of target genes may be significant to establishing the cancer risk of certain individual. However, to the best of our knowledge, no studies have yet demonstrated a link between genetic variations of the 3'UTR of the CDK6 gene and susceptibility of Chinese women to developing cervical precancerous lesions. In the present results, the minor A allele in CDK6-rs8179 reduced the risk of cervical precancerous lesions and had an antagonistic interaction with the HPV infection, as did the minor T allele in CDK6-rs42033. Considering than functional variations, such as SNPs located in the miRNA seed-match region, affect the binding affinity of miRNAs to target mRNAs, causing differential regulation of target gene expression and altering numerous molecular pathways simultaneously, rs8179 and rs42033 in the 3'UTR of the CDK6 gene may affect miRNA-mediated regulatory function. This leads to changes in the level of CDK6 expression, which may affect the development of cervical precancerous lesions. 
Table VII. Haplotype frequencies of CDK6 polymorphisms and cervical precancerous lesions.

Haplotype frequency

\begin{tabular}{lcccccc} 
& Control $(\%)$ & Cases $(\%)$ & OR $^{\mathrm{b}}(95 \% \mathrm{CI})$ & P-value & OR $^{\mathrm{b}, \mathrm{c}}(95 \% \mathrm{CI})$ & P-value $^{\mathrm{c}, \mathrm{e}}$ \\
\hline GAAG & 81.90 & 83.84 & 1.00 & - & 1.00 & - \\
GGAA & 10.46 & 13.72 & $1.29(0.85-1.98)$ & 0.240 & $1.67(0.99-2.84)$ & 0.057 \\
AGTA & 3.72 & 1.22 & $0.32(0.11-0.95)$ & 0.040 & $0.21(0.06-0.75)$ & 0.016 \\
GAAA & 1.71 & 0.92 & $0.64(0.17-2.46)$ & 0.520 & $0.45(0.10-2.13)$ & 0.320 \\
Rare $^{\mathrm{d}}$ & 1.54 & 0.31 & $0.21(0.03-1.53)$ & 0.120 & $0.17(0.02-1.49)$ & 0.110 \\
\hline
\end{tabular}

${ }^{a}$ CDK6 haplotypes, rs8179, rs4272, rs42033 and rs42377; b case vs. control group; cadjusted for HPV infection, age (years), body mass index

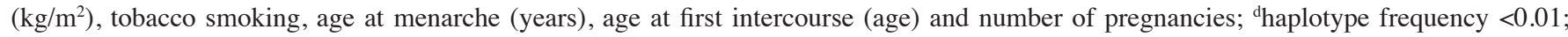
eglobal haplotype association $\mathrm{P}$-value, 0.0022 . The emboldened result is statistically significant $(\mathrm{P}<0.05)$. CDK6, cyclin-dependent kinase 6; OR, odds ratio; CI, confidence interval.

A
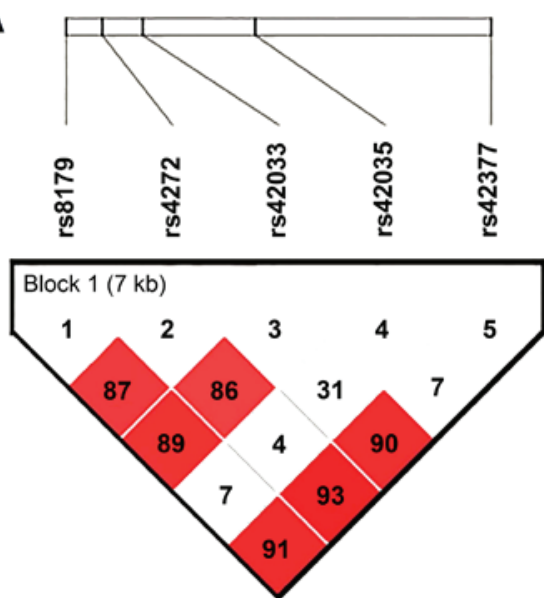

B
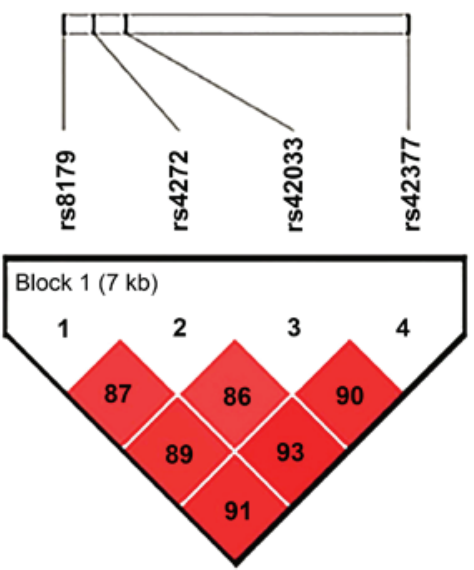

Figure 1. Linkage disequilibrium plots of (A) rs8179, rs4272, rs42033, rs42035, rs42377 and (B) rs8179, rs4272, rs42033, rs42377 in cyclin-dependent kinase 6 gene of the 460 study subjects.

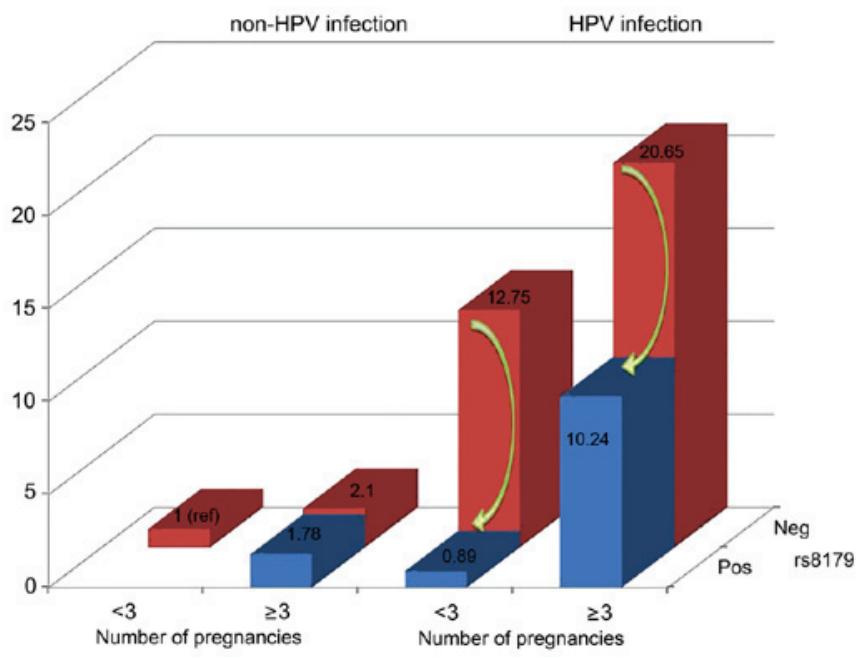

Figure 2. Risk analysis with three factors: Human papillomavirus infection, number of pregnancies and rs8179.

The underlying mechanism requires further investigation in functional studies.
In addition, one study indicated that an increased CDK6 expression level was significantly associated with the histological grade of cervical carcinogenesis and that CDK6 was positively associated with E6/E7 (10). The authors indicated that CDK6 may serve as a candidate HPV-induced oncogene in cervical cancer and HR-HPVs may gain an advantage in inducing carcinogenesis by reshaping the environment of the cellular miRNA composition and target gene expression to benefit the virus (10). Therefore, the present study hypothesized that there is an interaction between HPV and each SNP in the CDK6 gene, which is associated with the risk of cervical precancerous lesions. Additionally, the current study demonstrated an interactive effect between rs8179, rs42033 and the HPV infection on an additive scale. Furthermore, the MDR model indicated that the interaction between the HPV infection, rs8179 and the number of pregnancies was important at the population level; among HPV-infected individuals who had $<3$ pregnancies, women carrying the minor A allele in CDK6-rs8179 had a 50\% reduced risk of cervical precancerous lesions compared with individuals who were wild type at rs8179 (Fig. 2). Additionally, using haplotype analysis, it was identified that the haplotype AGTA was associated with 
a decreased risk of cervical precancerous lesions. In addition to the HPV infection, cervical precancerous lesions are associated with various environmental factors. The current results demonstrated that women with a smoking history, shorter TMI and higher number of pregnancies were at risk of cervical precancerous lesions. Smoking reduces cervical immunity, which enhances the persistence of the HPV infection (37). Various studies have demonstrated that women with cervical precancerous lesions had a shorter TMI $(38,39)$. It was hypothesized that this group may exhibit delayed maturation of the cervical transformation zone and, thus, an increased area of immature epithelium, suggesting that a subpopulation of adolescents with cervical precancerous lesions have a biologically immature cervix, which may increase the vulnerability of the cervical epithelium to HPV infection and neoplastic change may, therefore, increase the risk of cervical carcinoma, as it retains the transformation zone on the exocervix for many years, facilitating direct exposure to HPV (38-40).

There were various limitations of the current study. The sample size for genotyping was relatively small, resulting in a low statistical power of the genetic aspect of this study. Larger studies involving individuals from different ethnic populations are required to verify the findings, and this may facilitate the elucidation of the intrinsic mechanism. In addition, these data demonstrated the protective effect of the polymorphisms of the CDK6 gene in cervical precancerous lesions; however, the precise molecular mechanism underlying the effect remains to be elucidated, which would provide a basis for further studies (for example, where mutations are cloned in luciferase reporter constructs to characterize the binding specificity of miR-29). Protein expression studies of a similar group of patients are being considered to determine whether the polymorphisms are associated with cervical precancerous lesions.

In conclusion, the current data provide novel evidence that the rs8179 and rs42033 polymorphisms and haplotype AGTA in CDK6 may influence the development of cervical precancerous lesions in Chinese women. Furthermore, the present study demonstrates two- and three-way gene-environment interactions in the etiology of cervical precancerous lesions. Novel epidemiological clues regarding the protective role of the CDK6 gene in cervical precancerous lesions and further insights into the interactions involved in the etiology of cervical precancerous lesions were also presented. These results provide further evidence that introns should no longer be considered nonsense. In addition, the possibility that high risk HPVs may have an advantage in the induction of carcinogenesis in the environment of the cellular miRNA composition and target gene expression to the benefit of the virus.

\section{Acknowledgements}

The present study was supported in part by China Population Welfare Foundation (grant no. [2011]31), Science and Technology Planning Project of Guangdong Province (grant no. 2013B032000001), Family Planning Foundation of Guangdong Province (grant no. 2012004), National Natural Science Foundation of China (grant nos. 30901249, 81101267 and 81541070), the Guangdong Natural Science Foundation (grant nos. 10151063201000036 and S2011010002526),
Guangdong Province Medical Research Foundation (grant no. A2014374 and A2015310) and Project from Jinan university (grant no. 21612-426, 21615427). The authors would like to thank all of the collaborators, Yang Liu, Baohuan Zhang, Man Wang, Na Zhang and the staff for their hard work in running the study and the women who participated in this study.

\section{References}

1. Jemal A, Bray F, Center MM, Ferlay J, Ward E and Forman D: Global cancer statistics. CA Cancer J Clin 61: 69-90, 2011.

2. Torre LA, Bray F, Siegel RL, Ferlay J, Lortet-Tieulent J and Jemal A: Global cancer statistics, 2012. CA Cancer J Clin 65: 87-108, 2015.

3. Ambros RA and Kurman RJ: Current concepts in the relationship of human papillomavirus infection to the pathogenesis and classification of precancerous squamous lesions of the uterine cervix. Semin Diagn Pathol 7: 158-172, 1990.

4. Xiao D, Huang W, Ou M, Guo C, Ye X, Liu Y, Wang M, Zhang B, Zhang N, Huang S, et al: Interaction between susceptibility loci in cGAS-STING pathway, MHC gene and HPV infection on the risk of cervical precancerous lesions in Chinese population. Oncotarget 7: 84228-84238, 2016.

5. Nieves-Ramirez ME, Partida-Rodriguez O, Alegre-Crespo PE, Tapia-Lugo Mdel C and Perez-Rodriguez ME: Characterization of single-nucleotide polymorphisms in the tumor necrosis factor alpha promoter region and in lymphotoxin $\alpha$ in squamous intraepithelial lesions, precursors of cervical cancer. Transl Oncol 4: 336-344, 2011.

6. Bodily J and Laimins LA: Persistence of human papillomavirus infection: Keys to malignant progression. Trends Microbiol 19: 33-39, 2011.

7. Castle PE, Schiffman M, Wheeler CM and Solomon D: Evidence for frequent regression of cervical intraepithelial neoplasia-grade 2. Obstet Gynecol 113: 18-25, 2009.

8. Kobayashi A, Weinberg V, Darragh T and Smith-McCune K: Evolving immunosuppressive microenvironment during human cervical carcinogenesis. Mucosal Immunol 1: 412-420, 2008.

9. Zhang J, Wang L, Li B, Huo M, Mu M, Liu J and Han J: miR-145 downregulates the expression of cyclin-dependent kinase 6 in human cervical carcinoma cells. Exp Ther Med 8: 591-594, 2014.

10. Li Y, Wang F, Xu J, Ye F, Shen Y, Zhou J, Lu W, Wan X, Ma D and Xie X: Progressive miRNA expression profiles in cervical carcinogenesis and identification of HPV-related target genes for miR-29. J Pathol 224: 484-495, 2011.

11. Wang X, Meyers C, Guo M and Zheng ZM: Upregulation of p18Ink4c expression by oncogenic HPV E6 via p53-miR-34a pathway. Int J Cancer 129: 1362-1372, 2011.

12. Sridhar J, Akula N and Pattabiraman N: Selectivity and potency of cyclin-dependent kinase inhibitors. AAPS J 8: E204-E221, 2006.

13. Tadano T, Kakuta Y, Hamada S, Shimodaira Y, Kuroha M, Kawakami Y, Kimura T, Shiga H, Endo K, Masamune A, et al: MicroRNA-320 family is downregulated in colorectal adenoma and affects tumor proliferation by targeting CDK6. World J Gastrointest Oncol 8: 532-542,2016.

14. Lee AR, Park J, Jung KJ, Jee SH and Kim-Yoon S: Genetic variation rs7930 in the miR-4273-5p target site is associated with a risk of colorectal cancer. Onco Targets Ther 9: 6885-6895, 2016.

15. Zheng N, Yang P, Wang Z and Zhou Q: OncomicroRNAs-mediated tumorigenesis: Implication in cancer diagnosis and targeted therapy. Curr Cancer Drug Targets 17: 40-47, 2017.

16. Nayar R and Wilbur DC: The Pap test and Bethesda 2014. Cancer Cytopathol 123: 271-281, 2015.

17. Yi X, Li J, Yu S, Zhang A, Xu J, Yi J, Zou J, Nie X, Huang J and Wang J: A new PCR-based mass spectrometry system for high-risk HPV, part I: Methods. Am J Clin Pathol 136: 913-919, 2011.

18. Qu S, Huang J, Zhao J, Zhao X, Deng H, Yang H, Chen W, Liu L, Zhang L and Gao S: A comparison of matrix-assisted laser desorption/ionization time-of-flight mass spectrometry and surface plasmon resonance for genotyping of high-risk human papillomaviruses. Intervirology 54: 326-332, 2011.

19. Jurinke C, van den Boom D, Cantor CR and Köster H: Automated genotyping using the DNA MassArray technology. Methods Mol Biol 187: 179-192, 2002. 
20. Hahn LW, Ritchie MD and Moore JH: Multifactor dimensionality reduction software for detecting gene-gene and gene-environment interactions. Bioinformatics 19: 376-382, 2003.

21. Heidema AG, Feskens EJ, Doevendans PA, Ruven HJ, van Houwelingen HC, Mariman EC and Boer JM: Analysis of multiple SNPs in genetic association studies: Comparison of three multi-locus methods to prioritize and select SNPs. Genet Epidemiol 31: 910-921, 2007.

22. Tunesi S, Ferrante D, Mirabelli D, Andorno S, Betti M, Fiorito G, Guarrera S, Casalone E, Neri M, Ugolini D, et al: Gene-asbestos interaction in malignant pleural mesothelioma susceptibility. Carcinogenesis 36: 1129-1135, 2015.

23. Andersson T, Alfredsson L, Källberg H, Zdravkovic S and Ahlbom A: Calculating measures of biological interaction. Eur J Epidemiol 20: 575-579, 2005.

24. Muthuri SG, Doherty S, Zhang W, Maciewicz RA, Muir KR and Doherty M: Gene-environment interaction between body mass index and transforming growth factor beta 1 (TGF 31 ) gene in knee and hip osteoarthritis. Arthritis Res Ther 15: R52, 2013.

25. Knol MJ, van der Tweel I, Grobbee DE, Numans ME and Geerlings MI: Estimating interaction on an additive scale between continuous determinants in a logistic regression model. Int J Epidemiol 36: 1111-1118, 2007.

26. Knol MJ, VanderWeele TJ, Groenwold RH, Klungel $\mathrm{OH}$ Rovers MM and Grobbee DE: Estimating measures of interaction on an additive scale for preventive exposures. Eur $\mathbf{J}$ Epidemiol 26: 433-438, 2011.

27. Dai X, Li L, Liu X, Hu W, Yang Y and Bai Z: Cooperation of DLC1 and CDK6 affects breast cancer clinical outcome. G3 (Bethesda) 5: 81-91, 2014.

28. Xia B, Yang S, Liu T and Lou G: miR-211 suppresses epithelial ovarian cancer proliferation and cell-cycle progression by targeting Cyclin D1 and CDK6. Mol Cancer 14: 57, 2015.

29. Li LP, Wu WJ, Sun DY, Xie ZY, Ma YC and Zhao YG: miR-449a and CDK6 in gastric carcinoma. Oncol Lett 8: 1533-1538, 2014.

30. Lu X, Fang Y, Wang Z, Xie J, Zhan Q, Deng X, Chen H, Jin J, Peng C, Li H and Shen B: Downregulation of gas5 increases pancreatic cancer cell proliferation by regulating CDK6. Cell Tissue Res 354: 891-896, 2013.

31. Arvanitis DA and Spandidos DA: Deregulation of the G1/S phase transition in cancer and squamous intraepithelial lesions of the uterine cervix: A case control study. Oncol Rep 20: 751-760, 2008.

32. Choi YJ and Anders L: Signaling through cyclin D-dependent kinases. Oncogene 33: 1890-1903, 2014
33. Costello JF, Plass C, Arap W, Chapman VM, Held WA, Berger MS, Su Huang HJ and Cavenee WK: Cyclin-dependent kinase 6 (CDK6) amplification in human gliomas identified using two-dimensional separation of genomic DNA. Cancer Res 57: 1250-1254, 1997.

34. Zhou X, Chen X, Hu L, Han S, Qiang F, Wu Y, Pan L, Shen H, Li Y and Hu Z: Polymorphisms involved in the miR-218-LAMB3 pathway and susceptibility of cervical cancer, a case-control study in Chinese women. Gynecol Oncol 117: 287-290, 2010.

35. Reshmi G, Surya R, Jissa VT, Babu PS, Preethi NR, Santhi WS, Jayaprakash PG and Pillai MR: C-T variant in a miRNA target site of BCL2 is associated with increased risk of human papilloma virus related cervical cancer-an in silico approach. Genomics 98: 189-193, 2011.

36. Shi TY, Cheng X, Yu KD, Sun MH, Shao ZM, Wang MY, Zhu ML, He J, Li QX, Chen XJ, et al: Functional variants in TNFAIP8 associated with cervical cancer susceptibility and clinical outcomes. Carcinogenesis 34: 770-778, 2013.

37. Giuliano AR, Sedjo RL, Roe DJ, Harri R, Baldwi S, Papenfuss MR, Abrahamsen M and Inserra P: Clearance of oncogenic human papillomavirus (HPV) infection: Effect of smoking (United States). Cancer Causes Control 13: 839-846, 2002.

38. Syrjänen K, Shabalova I, Petrovichev N, Kozachenko V, Zakharova T, Pajanidi J, Podistov J, Chemeris G, Sozaeva L, Lipova E, et al: Age at menarche is not an independent risk factor for high-risk human papillomavirus infections and cervical intraepithelial neoplasia. Int J STD AIDS 19: 16-25, 2008

39. Moscicki AB, Winkler B, Irwin CE Jr and Schachter J: Differences in biologic maturation, sexual behavior, and sexually transmitted disease between adolescents with and without cervical intraepithelial neoplasia. J Pediatr 115: 487-493, 1989.

40. Muñoz N, Franceschi S, Bosetti C, Moreno V, Herrero R, Smith JS, Shah KV, Meijer CJ and Bosch FX; International Agency for Research on Cancer. Multicentric Cervical Cancer Study Group: Role of parity and human papillomavirus in cervical cancer: The IARC multicentric case-control study. Lancet 359: 1093-1101, 2002. 